\title{
Conductivity Tuning of a Silver Nanowire Mesh Using an UV Light
}

\author{
Shiyou Xu, Gerald Poirier, and Nan Yao \\ Princeton Institute for the Science and Technology of Materials (PRISM), Princeton University, \\ Princeton, NJ 08544 USA
}

Silver nanowire mesh based flexible, transparent and stretchable electrodes have attracted extensive attentions because they could be used for electrode applications in devices such as liquid crystal, flat panel or plasma displays, touch panels, organic light-emitting diodes (OLEDs), and solar cells, etc. Significant efforts have been paid for obtaining high conductivities and good stabilities. One practical way is to modify the connectivity between individual nanowires. Silver nanowires are often synthesized by a polymer assisted method, which uses molecules of the polymer to cap certain crystal faces of silver and cause silver growth only in the selective direction. When silver nanowires are made into a mesh, the residue polymer on the surface of the silver nanowire may form an isolation layer at the junctions of two nanowires. Therefore, the conductivity of the silver nanowire would increase if the residue polymer is removed. Further, if silver nanowires could be welded together, the conductivity should be much higher. A heating method using a laser has been used to treat and weld silver nanowire meshes, however, these methods need to be performed in a vacuum or a protective environment $[1,2]$. Here we present a simple technique to treat the silver nanowire mesh to increase the conductivity at an ambient condition.

Silver nanowires are synthesized by a modified method of Ref [2]. Figures 1A and 1B show typical SEM images of the silver nanowires synthesized at $110^{\circ} \mathrm{C}$. Large quantities of nanowires are produced. The diameter of the silver nanowires is about $100 \mathrm{~nm}$ and the length is up to $70 \mu \mathrm{m}$. Silvers nanowires are spin-casted between two iridium contact pads which are connected to a Keithley SCS4200 system. An UV light with a $254 \mathrm{~nm}$ wavelength is exposed on the silver nanowire mesh for certain amounts of time, and the I-V curves of the silver nanowire mesh are obtained. Figure 2 shows the I-V curves of the silver nanowire mesh at different treating time. The I-V curve shows rather linear relationships indicating that silver nanowires and the iridium contact pads have an ohmic contact. The resistances of the silver nanowire mesh are $1200 \Omega, 620 \Omega$ and $380 \Omega$, at no UV treatment, 1hour UV treatment and 12 hour treatment, respectively. These results prove that the UV light can increase the conductivity of the silver nanowire mesh at an ambient environment. One reason could be that the UV light covert the polymer on the surface of the silver nanowire to a conductive amorphous carbon which would increase the conductive contact area between two attached silver nanowires. The other reason could be that the UV light induces a joule heating at the contact area of two jointed silver nanowire, the two nanowires could be welded together at the junction. When silver nanowires stacks together, the small gaps that form naturally at nanowire junctions enable effective light concentration due to the plasmonic phenomena. Thus a local heating at the point where the wires joined together may be induced, and melt the nanowires to weld them together.

In summary, we have demonstrated a simple way to treat silver nanowire meshes to enhance their conductivity by expose to an UV light. The conductivity of a silver nanowire mesh almost tripled after a 12 hour UV treatment. The reason could be that a local heating at silver nanowire junctions generated by the plasmonic phenomena removes the polymer residues and weld nanowires at the junctions. The power of the UV light is $10 \mathrm{~W} / \mathrm{cm}^{2}$, a higher power UV source could work more 
efficiently. A detailed investigation of the contacting interface before and after UV treatment is underway.

\section{Reference:}

[1] J. Spechler, et al. Applied Physics A: Material Science and processing, 2012, online, DOI $10.1007 / \mathrm{s} 00339-012-6958-7$

[2] J. Lee, et al. Nanoscale 4(2012)6408.

[3] This work is supported in part by the National Science Foundation-MRSEC program through the Princeton Center for Complex Materials (DMR-0819860).
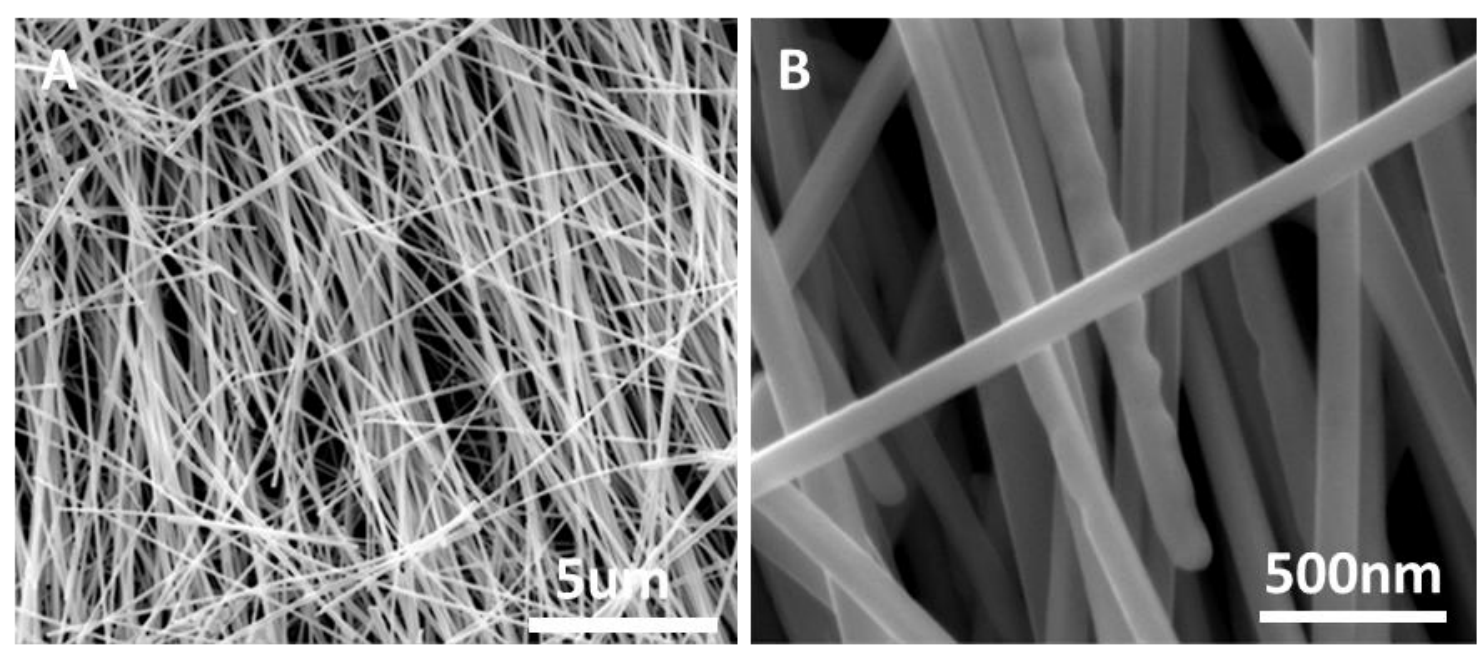

FIG. 1. SEM images of silver nanowires. A. Low magnification. B. High magnification.

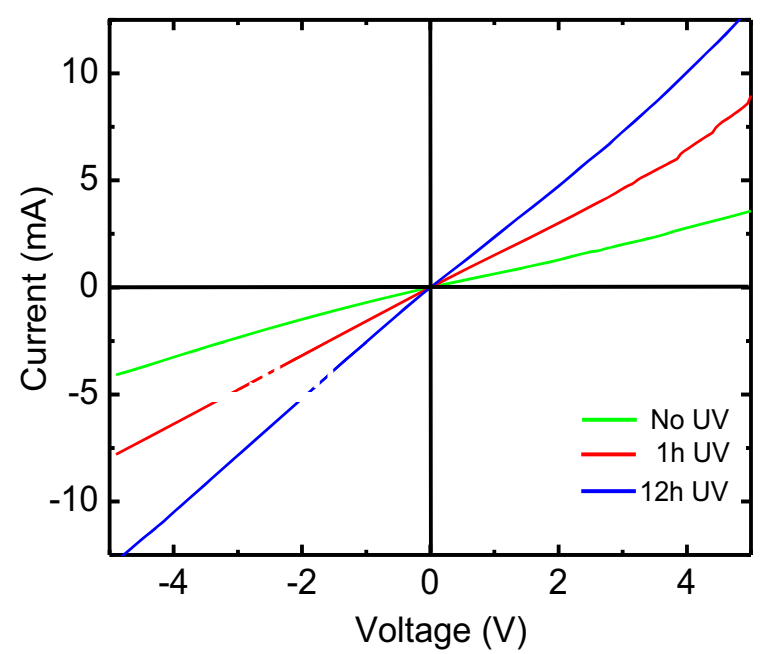

FIG. 2. I-V curves of the silver nanowire mesh treated at different time 